\title{
EPIDEMIOLOGISCHES PROFIL UND SOZIALER DETERMINANT VON COVID-19 IN MACAPA, AMAP, AMAZON, BRASILIEN
}

\section{ORIGINALER ARTIKEL}

SILVA, Anderson Walter Costa $^{1}$, CUNHA, Arthur Arantes ${ }^{2}$, ALVES, Giovana Carvalho ${ }^{3}$, CORONA, Rodolfo Antônio ${ }^{4}$, DIAS, Claudio Alberto Gellis de Mattos ${ }^{5}$, NASSIRI, Reza ${ }^{6}$, VEDOVELLI, Silvana7, VILHENA, Tania Regina Ferreira ${ }^{8}$, FAVACHO, Veronica Batista Cambraia ${ }^{9}$, SOUSA, Josiany Ferreira ${ }^{10}$, ARAÚJO, Maria Helena Mendonça ${ }^{11}$, OLIVEIRA, Euzébio ${ }^{12}$, DENDASCK, Carla Viana ${ }^{13}$, FECURY, Amanda Alves ${ }^{14}$

\footnotetext{
${ }^{1}$ Arzt, Spezialist für Management von Gesundheitssystemen und -diensten, Professor, Dozent und Forscher des Medizinstudiengangs am Campus Macapá, Föderale Universität Amapá (UNIFAP).

${ }^{2}$ Akademiker des Medizinischen Studiengangs Macapá Campus, Föderale Universität von Amapá (UNIFAP).

${ }^{3}$ Akademiker des Medizinischen Studiengangs Macapá Campus, Föderale Universität von Amapá (UNIFAP).

${ }^{4}$ Akademiker des Medizinischen Studiengangs Macapá Campus, Föderale Universität von Amapá (UNIFAP).

${ }^{5}$ Biologe, Doktor in Theorie und Verhaltensforschung, Professor und Forscher des Lizentiats in Chemie am Institut für grundlegende, technische und technologische Bildung von Amapá (IFAP).

${ }^{6}$ Arzt. Doktor der Hämatologie, Spezialist für Klinische Pharmakologie mit Globale Gesundheitsexpertise und Virusinfektionen. Professor in der Abteilung für Pharmakologie und Toxikologie und Familien- und Gemeinschaftsmedizin. Staatliche Universität von Michigan (MSU), Michigan, USA.

${ }^{7}$ Krankenschwester. Gesundheitsminister der Gemeinde Macapá AP (SESA AP).

${ }^{8}$ Krankenschwester. Master in Gesundheitswissenschaften. Städtisches Gesundheitsamt von Amapá (SESA AP).

${ }^{9}$ Krankenschwester. Doktortitel in Wissenschaft. Städtisches Gesundheitsamt von Amapá (SESA AP).

${ }^{10}$ Krankenschwester. Städtisches Gesundheitsamt von Amapá (SESA AP).

${ }^{11}$ Doktor, Master in Lehr- und Gesundheitswissenschaften, Professor, Lehrer und Forscher des Medizinischer Kurs am Macapá-Campus, Bundesuniversität Amapá (UNIFAP).

${ }^{12}$ Biologe, Promotion in Tropenkrankheiten, Professor und Forscher des Sportunterricht bei Bundesuniversität Pará (UFPA).

${ }^{13}$ Theologe, PhD in Psychoanalyse, Forscher am Zentrum für Forschung und fortgeschrittene Studien - CEPA.

${ }^{14}$ Biomedizin, PhD in Topischen Krankheiten, Professor und Forscher des Medizinischer Kurs am MacapáCampus, Bundesuniversität Amapá (UNIFAP).
}

RC: 71076

Verfügbar in: https://www.nucleodoconhecimento.com.br/gesundheit/von-covid-19-inmacapa 
SILVA, Anderson Walter Costa. Et al. Epidemiologisches Profil und sozialer Determinant von COVID-19 in Macapa, Amap, Amazon, Brasilien. Revista Científica Multidisciplinar Núcleo do Conhecimento. Jahrgang 05, Ed. 04, Vol. 04, S. 05-27. April 2020. ISSN: 2448-0959, Zugangslink: https://www.nucleodoconhecimento.com.br/gesundheit/von-covid-19-in-macapa, DOI: 10.32749/nucleodoconhecimento.com.br/gesundheit/von-covid-19-in-macapa

\section{ZUSAMMENFASSUNG}

Ende Dezember 2019 tauchte in Wuhan, China, eine neue Vielfalt von Coronaviren auf, die von der Weltgesundheitsorganisation (WHO) zum globalen Gesundheitsnotstand erklärt wurde. Atemwegstropismus kann auf die Expression von Angiotensin-zu-Konverter-Enzym 2 (ACE2) zurückzuführen sein. In der Region der brasilianischen Legal Amazon, bis zum 22. März 2020, der Staat Amazonas präsentiert 26 bestätigte Fälle; Acre 11 Fälle; Para de 04; Rondonia 03; Roraima, Tocantins, Maranhéo und Mato Grosso 02 Fälle; und Amap nur 01, wenn bestätigt. Diese Studie zielt darauf ab, die ersten vermuteten und bestätigten Fälle von COVID19 in Macapa, Amapa, Amazon, Brasilien zu quantifizieren und zu analysieren. Dabei handelt es sich um eine beobachtungs-, retrospektive und quantitative Studie, die sich auf das Profil der ersten 108 Verdachtsfälle bezieht, die zwischen dem 13. März 2020 und dem 21. März 2020 in Macapa gemeldet wurden, und auf die Berechnungen der Inzidenz von COVID-19 in den 26 brasilianischen Hauptstädten und in Brasilia, Federal District, zwischen dem 26. Februar 2020 und dem 26. März 2020. Die Studien können ein spezifisches Muster der Verbreitung des Virus aufdecken, das zur Planung und Durchführung wirksamerer epidemiologischer Kontroll- und Überwachungsmaßnahmen beitragen würde.

Schlüsselwörter: COVID 19, ACE2, Coronavirus, Pandemie.

RC: 71076

Verfügbar in: https://www.nucleodoconhecimento.com.br/gesundheit/von-covid-19-inmacapa 


\section{EINFÜHRUNG}

Ende Dezember 2019 tauchte in Wuhan, China, eine neue Sorte von Coronavirus auf, das in der Lage ist, ein schweres akutes Atemwegssyndrom (SRHS) zu produzieren, und wurde von der Weltgesundheitsorganisation zum globalen Gesundheitsnotstand erklärt. Dieses Virus, genannt SARS-CoV-2, produziert eine Krankheit, die als COVID-19 (ICD-10-B34.2) (BRASIL, 2020a; WHO, 2020a; MCINTOSH, 2020). SARS-CoV-2 gehört zur Gattung betaCovs, der Unterfamilie Orthocoronavirinae, aus der Familie der Coronaviridae. Es ist ein Envelopado-Virus (eine doppelte Lipidmembran mit in sie eingeführten Proteinen) von einbandgebundener RNA, die aufgrund der spindlyförmigen Glykoproteine in ihrer Hülle die Form einer Krone hat (CASCELLA et al., 2020; VELAVAN et al., 2020).

Die häufigsten, aber unspezifischen Symptome im Zusammenhang mit einer Infektion durch dieses Virus sind: Fieber (83,0\%-99,0\%), trockener Husten (59,4\%82,0\%), Dyspnoe (55,0\%), Müdigkeit (Müdigkeit) (38,1\%), Halsschmerzen (13,9\%), Kopfschmerzen (Kopfschmerzen) (13,6\%) und Durchfall (3,7\%) (BRASILIEN, 2020a; LI et al., 2020; WHO, 2020a). Diese Symptome können im Einklang mit der Inkubationszeit des Virus auftreten, im Durchschnitt, 5 bis 6 Tage nach der Infektion (WHO, 2020a; ROTHAN et al., 2020).

Der Tropismus der Atemwege kann auf die Expression des Angiotensin-KonverterEnzyms 2 (ACE2) im Pulmonalenparenchym, dem menschlichen Atemwegsepithel und dem vaskulären Endothel zurückzuführen sein. ACE2 ist einer der Hauptvermittler des Eindringens des Virus in die Zellen des menschlichen Wirts, der als Rezeptor für den Eintrag von Krankheitserregern fungiert. Dieser Mechanismus reicht jedoch nicht aus, um die Beteiligung menschlicher Zelllinien zu erklären, die nicht ACE2 ausdrückten, aber infiziert waren (LI et al., 2020; LI et al., 2003; ROTHAN et al., 2020).

RC: 71076

Verfügbar in: https://www.nucleodoconhecimento.com.br/gesundheit/von-covid-19-inmacapa 
Ein Merkmal von SARS-CoV-2, das dazu beigetragen hat, dass es zu einem globalen Problem der öffentlichen Gesundheit wurde, ist sein hohes Übertragungspotenzial, das durch Femitis (kontaminierte unbelebte Materialien, die als Übertragungsvehikel dienen) und Atemtröpfchen von Husten und/oder Niesen (Aerosolübertragung) auftritt. Diese Übertragung kann, abhängig von der Konzentration von Viruspartikeln in der Umwelt, und die virale Lebensfähigkeit in der Aerosoldispersion wurde für drei Stunden oder mehr nachgewiesen. Der orofecalWeg scheint nicht wirksam zu sein, obwohl in einigen Fällen lebensfähige Viren gefunden wurden (CASCELLA, et al., 2020; DOREMALEN, et al., 2020; WHO, 2020a). SARS-Cov-2 ist nach SARS und MERS die dritte globale Bedrohung für die öffentliche Gesundheit (NASSIRI, 2020).

Nach Angaben des Notfallzentrale des öffentlichen Gesundheitswesens (COE-nCoV) sind die grundlegenden Präventionsmaßnahmen: Hände waschen häufig mit Seife und Wasser (für mindestens 20 Sekunden); in der Unmöglichkeit der Verwendung von Wasser und Seife, als alternativer Alkohol 70\% oder ein alkoholfreies Desinfektionsmittel verwenden; vermeiden Berührungen auf gesicht, Augen und Mund mit ungewaschenen Händen; Abdeckung mit Taschentuch Mund und Nase beim Niesen oder Husten (und dann den Schal entsorgen); häufig berührte Objekte und Oberflächen (Türklinke, Mobiltelefon, Fernbedienung); Kontakt mit Kranken vermeiden (Entzug); und bleiben Sie zu Hause, wenn Sie Symptome auftreten, auch wenn häufig Grippe (BRASIL, 2020b).

Die Einleitung des Protokolls zur Behandlung von COVID Pneumonia (2019-nCoV) hängt von der frühzeitigen Erkennung der Zeichen ab, die kontinuierlich überwacht werden, unter Berücksichtigung der klinischen Manifestationen und der allgemeinen Merkmale der Infektion. Dazu hat das brasilianische Gesundheitsministerium ein Behandlungsprotokoll (BRASIL, 2020b; BRASIL, 2020c).

Die hohe Übertragungsrate in Verbindung mit dem derzeitigen Fehlen eines spezifischen antiviralen Medikaments zur Behandlung (BRASIL, 2020c)

$\mathrm{RC}: 71076$

Verfügbar in: https://www.nucleodoconhecimento.com.br/gesundheit/von-covid-19-inmacapa 
vorausgesetzt, die 55.924 Fälle von COVID-19 wurden in China bis zum 20. Februar 2020 bestätigt (WHO, 2020b). Tödlichkeit von 44.672 (79,9\%) variierte je nach Altersgruppe, wobei $0,2 \%$ bei Patienten im Alter von 10-39 Jahren und 8,0\% bei Patienten im Alter von $70-79$ Jahren und $14,8 \%$ bei Patienten im Alter von $\geq 80$ Jahren, der am stärksten gefährdeten Gruppe, erreicht wurden. Die Variation der Letalitätsrate kann durch damit verbundene Risikofaktoren wie frühere Kardiovaskuläre Seuchen, Diabetes, Bluthochdruck, chronische Atemwegserkrankungen und Krebs (GALLASCH et al., 2020; ZHANG et al., 2020; WHO, 2020a). In Italien, dem zweiten Land mit der höchsten Beteiligung, wurden bis zum 20. März 2020 53.578 Fälle von COVID-19 mit 4.827 Todesfällen bestätigt, was zu einer allgemeinen Tödlichkeit von 9,0\% führte (WHO, 2020b).

In Brasilien wurde der erste Fall von COVID-19 am 26. Februar 2020 (WHO, 2020c) bestätigt und bis zum 22. März 2020 wurden 1.546 Fälle und 25 Todesfälle durch COVID-19 bestätigt (22 im Bundesstaat Sao Paulo und 03 im Bundesstaat Rio de Janeiro). Davon sind 926 (59,9\%) wurden in der Region Südosten aufgezeichnet; 231 (14,9\%) in der Region Nordosten; 179 (11,6\%) in der Region Süd; 161 (10,4\%) im Mittleren Westen; und 49 (3,2\%) in der nördlichen Region des Landes (BRASIL, 2020d).

In der Region Nord gab es im Bundesstaat Amazonas bis zum selben Zeitpunkt 26 bestätigte Fälle; die Fälle von Acre 11; Abs. 04; Rondônia 03; Fälle von Roraima und Tocantins 02; und Amapá nur 01 bestätigter Fall (BRASILIEN, 2020d).

Angesichts des exponentiellen Anstiegs der Fälle von COVID-19 und der Herausforderungen für die öffentliche Gesundheit Brasiliens sind epidemiologische Studien, die die Besonderheiten der betroffenen Bevölkerung bewerten und den Einfluss sozialer Faktoren auf die Übertragbarkeitsraten des Virus analysieren, äußerst wichtig und von sanitärem Bedarf (LIPSITCH et al., 2020).

RC: 71076

Verfügbar in: https://www.nucleodoconhecimento.com.br/gesundheit/von-covid-19-in$\underline{\text { macapa }}$ 


\section{TOR}

Quantifizierung und Analyse des ursprünglichen Index der vermuteten und anschließend bestätigten Fälle von COVID-19 in Macapá, Amapá, Amazon, Brasilien.

Um die Inzidenz von bestätigten Fällen von COVID-19 in Hauptstädten im legalen Amazonas im Vergleich zu anderen brasilianischen Hauptstädten zu analysieren und korrelieren mit sozioökonomischen, soziodemografischen und Gesundheitsindikatoren.

\section{MATERIALIEN UND METHODEN}

\section{STUDIENZEITEN}

Dies ist eine Beobachtungs-, Retrospektive- und quantitative Studie. Diese Studie hat zwei Zeiträume. Einer von ihnen, der sich auf das Profil der ersten 108 Verdachtsfälle bezieht, die in Macapa gemeldet wurden, ist zwischen dem 13. März 2020, dem Datum der Meldung des ersten Verdachtsfalls in Brasilien (WHO, 2020c), und dem 21. März 2020 aufgrund der Verordnung Nr. 454 des Gesundheitsministeriums (MS), die den Zustand der Übertragung in Brasilien erklärt hat (BRASIL, 2020e), begrenzt. Es sei darauf hingewiesen, dass Fälle, die bis zum 21. März gemeldet wurden, aufgrund der Frist für die Laboranalyse einige Tage nach der Anmeldung ihre Bestätigung/Entsorgung erhalten können.

Der andere Zeitraum dieser Studie, der sich auf die Berechnungen der Inzidenz von COVID-19 in den 26 brasilianischen Hauptstädten und Brasília, Federal District, bezieht, ist zwischen dem 26. Februar 2020, dem Datum des ersten bestätigten Falls in Brasilien (WHO, 2020c), und dem 26. März 2020, einen Monat nach der Bestätigung des ersten Falles, begrenzt.

RC: 71076

Verfügbar in: https://www.nucleodoconhecimento.com.br/gesundheit/von-covid-19-inmacapa 


\section{DATEN, VARIABLEN UND BERECHNUNGSMETHODEN}

In dieser Studie wurden sekundäre Daten verwendet. Die Daten über das Profil der ersten 108 Verdachtsfälle in Macapá wurden aus der Datenbank und den aggregierten Informationen extrahiert, die vom Gesundheitsministerium der Gemeinde Macapá, Amapá, Brasilien, entwickelt wurden.

Die Bevölkerungsdaten der Gemeinde Macapá wurden anhand der Nationalen Erhebung nach kontinuierlicher Haushaltsstichprobe 2016-2018 (BRASIL, 2018a) geschätzt, wobei die Bevölkerung von Macapá im Jahr 2019 bezugsweise (BRASIL, 2020f) stammt. Diese Bevölkerungsdaten wurden bei der Berechnung des Verdachtsfallkoeffizienten (CCS) nach Altersgruppen unabhängig vom Geschlecht verwendet, die nach der Formel berechnet wurden:

$$
\text { Coeficiente }(\mathrm{CCS})=\frac{\text { Número de casos suspeitos em determinada faixa etária } \times 100.000}{\text { Quantitativo populacional estimado da faixa etária referida }}
$$

Die quantitativ bestätigten Fälle von COVID-19, die zur Berechnung der Inzidenz (pro 100.000 Einwohner) in brasilianischen Hauptstädten bis zum 26. März 2020 verwendet wurden, wurden der Coronavirus-Karte (2020) entnommen, die offizielle und aktualisierte aggregierte Daten aller brasilianischen Gemeinden mit bestätigten Fällen liefert, die von den staatlichen Gesundheitsämtern zur Verfügung gestellt werden. Andererseits wurden die bei der Berechnung der Inzidenzverwendeten verwendeten Bevölkerungsdaten aus IBGE - Städte und Staaten extrahiert (BRASIL, 2020f). Die Methode zur Berechnung der Inzidenzen war:

$$
\text { Incidência }=\frac{\text { Número de casos confirmados em determina capital } \times 100.000}{\text { Quantitativo populacional da capital referida, no ano de } 2019}
$$

RC: 71076

Verfügbar in: https://www.nucleodoconhecimento.com.br/gesundheit/von-covid-19-inmacapa 
Sozioökonomische Indikatoren des Human Development Index (HDI) und ein Prozentsatz armer Menschen wurden der Atlas Brasil-Plattform (2020) entnommen. Die Anzahl der Gesundheitseinrichtungen (Bezugszeitraum: Oktober 2015) wurde der DATASUS-Plattform (BRASIL, 2020g) des Gesundheitsministeriums (MS) entnommen. Die demografische Dichte (DD) wurde anhand aktueller Informationen berechnet, da sich der letzte Beamte auf die IBGE-Volkszählung 2010 bezieht. So wurden die Daten der territorialen Fläche der Hauptstadt (Referenzjahr: 2018) und der Kapitalbevölkerung (Referenzjahr: 2019) zur Berechnung von DD (DD = Kapitalbevölkerung im Jahr 2019 / Hauptstadtgebiet in Quadratkilometern im Jahr 2018) verwendet.

\section{AUFNAHME- UND AUSSCHLUSSKRITERIEN VON DATEN}

Für die Analyse des Geschlechts der 108 Erstaufzeichnungen wurden alle Verdachtsfälle $(n=108)$ eingeschlossen; ab dem Zeitpunkt zwischen den ersten Anzeichen/Symptomen und der Meldung des Falls wurden acht Fälle ausgeschlossen, davon fünf Männer und drei Frauen $(n=100)$; für Alter, Wohnsitzland, Anzahl der Anzeichen/Symptome und Vorerkrankungen wurde nur ein weiblicher Fall ausgeschlossen ( $n=107)$; bezüglich Reisen außerhalb Brasiliens und Kontakt mit einem Verdachts- oder bestätigten Fall wurden drei Fälle ausgeschlossen, alle weiblich ( $n=105)$; bezüglich der berichtenden Gesundheitsstelle wurden 12 Fälle ausgeschlossen ( $n=96)$; bezüglich Beruf wurden 3 Fälle ausgeschlossen $(n=105)$. Alle Ausschlüsse waren auf die Nichtexistenz der Informationen zurückzuführen ("ignoriert"). Die "Ausreißer" (abweichende Werte) wurden nicht aus der Analyse ausgeschlossen.

In die Analyse der Inzidenzen und Korrelationen wurden alle 26 Hauptstädte der brasilianischen Föderativen Einheiten und Brasilia, Bundeshauptstadt, einbezogen. Die Hauptstädte wurden in zwei Gruppen eingeteilt, von denen eine aus den brasilianischen Hauptstädten im brasilianischen legalen Amazonasgebiet (Belém, Boa Vista, Cuiabá, Macapá, Manaus, Palmas, Porto Velho, Rio Branco und São

RC: 71076

Verfügbar in: https://www.nucleodoconhecimento.com.br/gesundheit/von-covid-19-inmacapa 
Luís) (BRASIL, 2014) und die anderen von den übrigen 17 Hauptstädten plus der Bundeshauptstadt besteht. Die Gesamtzahl der bestätigten Fälle bis 21 Stunden und 6 Minuten am 26. März 2020 wurde zur Berechnung der Inzidenz einbezogen. Ausreißer wurden von der Analyse nicht ausgeschlossen.

\section{STATISTISCHE ANALYSE}

Die Daten wurden mit Microsoft Exce ${ }^{\circledR} 2016$ Software, OriginPro ${ }^{\circledR}$ Software Version 8.0724 und Statistical Package for the Social Sciences ${ }^{\circledR}$ Version 20.0 Software organisiert, tabelliert und analysiert. Bemerkenswert ist, dass die verwendete OriginPro ${ }^{\circledR}$-Software das Einfügen eines scharfen Akzents in die Grafiken nicht akzeptierte.

Die Datenverteilung, falls relevant, wurde mit den Kolmogorov-Smirnov- und ShapiroWilk-Tests getestet. Die Auswahl des Tests erfolgte nach dem Stichprobenumfang (TORMAN et al., 2012). Die Varianzhomogenität wurde, falls relevant, mit dem mittelbasierten Levene-Test (LEVIN et al., 2018).

Für die statistische Analyse der ersten 108 Verdachtsfälle wurde der $t$-Test des Student verwendet, um die Mittel der unabhängigen Proben oder den Mann-Whitney U-Test auf mediane Differenz zu differenziert. Variablen, deren Hypothese der Normalverteilung nicht abgelehnt wurde und die eine Homogenität der Varianz aufwiesen, wurden mit dem $t$-Test getestet, während die Variablen, die diese Anforderungen nicht erfülten, mit dem Mann-Whitney-Test getestet wurden (TORMAN et al., 2012). Wir analysierten, verglichen die Geschlechter, die signifikante Existenz von Unterschieden des Mittleren Oder des mittleren Alters (in Jahren), das Intervall der Tage zwischen dem ersten Zeichen/Symptom bis zum Datum der Meldung des Verdachtsfalls und die quantitative Anzahl der Anzeichen/Symptome dargestellt. Das Signifikanzniveau betrug $p$-Wert $\leq 0,05$.

RC: 71076

Verfügbar in: https://www.nucleodoconhecimento.com.br/gesundheit/von-covid-19-inmacapa 
Die Analyse des Inzidenzunterschieds zwischen den Hauptstädten in der brasilianischen Legal Amazon Assregion $(n=9)$ und den anderen brasilianischen Hauptstädten ( $n=18)$ wurde ohne Berücksichtigung der Hypothese der Normalverteilung durchgeführt, da die Stichprobengröße kleiner als 10 ist. So wurde die nicht-parametrische Alternative der Analyse, Mann-whitney u test, durchgeführt. Das Signifikanzniveau betrug $p$-Wert $\leq$ 0,05 (LEVIN et al., 2018; TORMAN et al., 2012).

Die Hypothese der Normalverteilung wurde für die folgenden Variablen nicht zurückgewiesen: Inzidenz brasilianischer Hauptstädte, HDI der brasilianischen Hauptstädte, Prozentsätze armer Menschen in brasilianischen Hauptstädten und Quantitative von Gesundheitseinrichtungen in brasilianischen Hauptstädten. So wurde der Pearson-Korrelationstest (bivariat) fortgesetzt. Die Richtungen der Korrelationen (positiv oder negativ) und die Intensität des PearsonKorrelationskoeffizienten $(r)$ wurden beobachtet. Die Intensität (stark, mäßig, schwach oder nicht vorhanden) wird nach $r$-Wert (LEVIN et al., 2018). Das signifzintherspezifische Signifikanzniveau betrug den $p$-Wert $\leq 0,05$.

Es wurden beschreibende Statistiken berechnet: Mittelwert $(\bar{x})$, Standardabweichung, Median, Minimum und Maximum.

\section{EINSCHRÄNKUNGEN}

Zu den Einschränkungen dieser Studie gehören Fälle, die nach den Richtlinien der MS (BRASIL, 2020h) nicht als Verdächtige gemeldet werden sollten, die aber die untersuchte Stichprobe bilden. Und das Fehlen einiger Informationen in der Datenbank (z. B. Beruf, Datum des ersten Symptoms und Benachrichtigung der Gesundheitseinheit), wahrscheinlich aufgrund eines falschen oder unvollständigen Ausfüllens des Anmeldeformulars.

$\mathrm{RC}: 71076$

Verfügbar in: https://www.nucleodoconhecimento.com.br/gesundheit/von-covid-19-inmacapa 


\section{ETHISCHE ASPEKTE}

Die Studie verwendete aggregierte Sekundärdaten, die keine individuelle Identifizierung zulassen, die nach institutioneller Vereinbarung in der Datenbank des städtischen Gesundheitsamtes von Macapá, Amapá, Amazon, Brasilien, verfügbar sind. Zusätzlich zu den Informationen, die unter elektronischen Adressen mit offenem Zugang verfügbar sind. Diese Forschung folgte den Kriterien der Resolutionen Nr. 466/2012 und Nr. 510/2016 des brasilianischen Nationalen Rates für Forschungsethik (CONEP).

\section{ERGEBNISSE}

\section{ANALYSE DER ERSTEN 108 SUSPECTED CASES IN MACAP}

Von den 108 in dieser Studie analysierten Verdachtsfällen, die zwischen dem 13. März 2020 und dem 21. März 2020 gemeldet wurden, sind drei (2,78\%) wurden durch Molekülanalyse (RT PCR) mit COVID-19 bestätigt. Alle waren Brasilianer, zwei weibliche Fälle (66,66\%) und eines der Männer (33,33\%). Das Durchschnittsalter der drei Fälle betrug 36,3 Jahre. Die drei (100,0\%) Fieber, Kopfschmerzen, laufende Nase. Zwei $(66,66 \%)$ Husten, Sputumproduktion und Atembeschwerden hatten. Alle drei machten eine Reise aus Brasilien in den 14 Tagen vor dem Datum der Benachrichtigung. Die mittlere Zeit der drei bestätigten Fälle zwischen dem Datum des ersten Vorzeichens/Symptoms und dem Datum der Meldung betrug 3 Tage.

Das Profil der Gesamtzahl der analysierten Verdachtsfälle war wie folgt: $(n=107$; $99,07 \%$ ), weiblich ( $n=59 ; 54,62 \%)$, Altersgruppe 30-39 Jahre ( $n=29 ; 27,1 \%)$, ohne vorherige Morbiditäten ( $n=78 ; 72,22 \%)$ (Tabelle 1 bzw. Tabelle 2). Das Durchschnittsalter (einschließlich beider Geschlechter) betrug $\bar{x}=34,2 \pm 14,9$, mit einem Median von 34 Jahren. Es gab keinen signifikanten Unterschied im Mittel zwischen den Geschlechtern ( $t=0,331 ; p=0,742)$ (Tabelle 3).

RC: 71076

Verfügbar in: https://www.nucleodoconhecimento.com.br/gesundheit/von-covid-19-inmacapa 
Tabelle 1 Häufigkeit und Prozentsatz der analysierten Verdachtsfälle von COVID-19 und Koeffizienten nach Altersgruppen nach Geschlecht und Altersgruppe. Macapá, Amapá, 13.-21. März 2020.

\begin{tabular}{|c|c|c|c|c|c|c|}
\hline \multirow{2}{*}{$\begin{array}{c}\text { Faixa Etária } \\
\text { (anos) }\end{array}$} & \multirow{2}{*}{$\begin{array}{c}\text { Masculino } \\
\text { n (\%) }\end{array}$} & \multirow{2}{*}{$\begin{array}{c}\text { Feminino } \\
\mathbf{n}(\%)\end{array}$} & \multicolumn{2}{|c|}{ Total } & \multirow{2}{*}{$\begin{array}{l}\text { *População } \\
\text { Estimada }\end{array}$} & \multirow{2}{*}{$\begin{array}{c}* \text { Coeficiente } \\
\text { (por } 100.000 \text { pessoas) }\end{array}$} \\
\hline & & & $\mathbf{n}$ & $\%$ & & \\
\hline $0-19$ & $7(54 \%)$ & $6(46 \%)$ & 13 & $12,1 \%$ & 174.395 & 7,45 \\
\hline $20-29$ & $11(41 \%)$ & $16(59 \%)$ & 27 & $25,2 \%$ & 90.708 & 29,77 \\
\hline $30-39$ & $17(59 \%)$ & $12(41 \%)$ & 29 & $27,1 \%$ & 74.386 & 38,99 \\
\hline $40-49$ & $7(30 \%)$ & $16(70 \%)$ & 23 & $21,5 \%$ & 73.120 & 31,46 \\
\hline $50-59$ & $4(40 \%)$ & $6(60 \%)$ & 10 & $9,3 \%$ & 42.460 & 23,55 \\
\hline$\geq 60$ & $3(60 \%)$ & $2(40 \%)$ & 5 & $4,7 \%$ & 48.258 & 10,36 \\
\hline Total & $46(45 \%)$ & $57(55 \%)$ & $107 * * *$ & $100,0 \%$ & 503.327 & 21,26 \\
\hline
\end{tabular}

*Bevölkerung, die je nach Altersgruppe unabhängig vom Geschlecht geschätzt wird, für die Gemeinde Macapa; **Koeffizient (CCS) = Gesamt $n \times 100.000$ / Geschätzte Bevölkerung für das Alter; Ein weiblicher Fall hatte den Punkt "Alter" ignoriert.[/caption]

RC: 71076

Verfügbar in: https://www.nucleodoconhecimento.com.br/gesundheit/von-covid-19-inmacapa 
Tabelle 2 Häufigkeit und Prozentsatz früherer Morbiditäten von Verdachtsfällen von COVID-19 analysiert. Macapá, Amapá, 13.-21. März 2020.

Tabela 2 Frequência e porcentagem das morbidades prévias dos casos suspeitos de COVID-19 analisados. Macapá, Amapá, 13 a 21 de Março de 2020.

\begin{tabular}{|c|c|c|c|c|c|c|}
\hline \multirow{2}{*}{ Morbidade Prévia } & \multicolumn{2}{|c|}{ Masculino } & \multicolumn{2}{|c|}{ Feminino } & \multicolumn{2}{|c|}{ Total } \\
\hline & $\mathbf{n}$ & $\%$ & $\mathbf{n}$ & $\%$ & $\mathbf{n}$ & $\%$ \\
\hline $\begin{array}{l}\text { Doença cardiovascular, } \\
\text { incluindo hipertensão }\end{array}$ & 5 & $38,5 \%$ & 8 & $61,5 \%$ & 13 & $100,0 \%$ \\
\hline Doença pulmonar crônica & 3 & $50,0 \%$ & 3 & $50,0 \%$ & 6 & $100,0 \%$ \\
\hline Diabetes & 3 & $60,0 \%$ & 2 & $40,0 \%$ & 5 & $100,0 \%$ \\
\hline Doença hepática & 0 & $0,0 \%$ & 1 & $100,0 \%$ & 1 & $100,0 \%$ \\
\hline Neoplasia & 1 & $100,0 \%$ & 0 & $0,0 \%$ & 1 & $100,0 \%$ \\
\hline Imunodeficiência & 1 & $100,0 \%$ & 0 & $0,0 \%$ & 1 & $100,0 \%$ \\
\hline Doença neurologica crônica & 1 & $100,0 \%$ & 0 & $0,0 \%$ & 1 & $100,0 \%$ \\
\hline $\begin{array}{l}\text { Doença hepática e Doença } \\
\text { neuromuscular e Diabetes }\end{array}$ & 0 & $0,0 \%$ & 1 & $100,0 \%$ & 1 & $100,0 \%$ \\
\hline $\begin{array}{l}\text { Doença hepática e Doença } \\
\text { renal }\end{array}$ & 1 & $100,0 \%$ & 0 & $0,0 \%$ & 1 & $100,0 \%$ \\
\hline Total & 15 & $50,0 \%$ & 15 & $50,0 \%$ & 30 & $100,0 \%$ \\
\hline
\end{tabular}

RC: 71076

Verfügbar in: https://www.nucleodoconhecimento.com.br/gesundheit/von-covid-19-in$\underline{\text { macapa }}$ 
Tabelle 3 Ergebnisse statistischer Tests von Verdachtsfällen von COVID-19 analysiert. Macapá, Amapá, 13.-21. März 2020.

\begin{tabular}{|c|c|c|c|c|c|c|c|c|c|c|}
\hline \multirow{3}{*}{ Variável } & \multicolumn{6}{|c|}{ Sexo } & \multicolumn{4}{|c|}{ p-valor Testes Estatísticos } \\
\hline & \multicolumn{3}{|c|}{ Masculino } & \multicolumn{3}{|c|}{ Feminino } & \multirow{2}{*}{$\begin{array}{c}\text { p-valor } \\
\text { normalidade } \\
\text { (Masc./Fem.) }\end{array}$} & \multirow{2}{*}{$\begin{array}{l}\mathrm{p} \text {-valor } \\
\text { teste de } \\
\text { Levene }\end{array}$} & \multirow{2}{*}{$\begin{array}{l}\text { p-valor } \\
\text { teste } t \text { de } \\
\text { Student }\end{array}$} & \multirow{2}{*}{$\begin{array}{l}\text { p-valor U } \\
\text { Mann- } \\
\text { Whitney }\end{array}$} \\
\hline & Média $\pm \mathrm{DP}$ & Mediana & Máx/Min. & Média $\pm \mathrm{DP}$ & Mediana & Máx/Min. & & & & \\
\hline Idade (em anos) & $33,6 \pm 15,0$ & 34,0 & $67 / 0,4$ & $34,6 \pm 14,9$ & 35,5 & $78 / 1$ & $0,64 / 0,51$ & 0,809 & 0,741 & -- \\
\hline $\begin{array}{l}\text { Quantitativo de } \\
\text { Sinais/Sintomas } \\
\text { apresentados }\end{array}$ & $5,8 \pm 2,7$ & 5,0 & $12 / 1$ & $6,6 \pm 4,0$ & 5,0 & $20 / 1$ & $0,44 / 0,053$ & 0,037 & $-\cdots$ & 0,488 \\
\hline $\begin{array}{l}\text { Intervalo de dias: } \\
1^{\circ} \text { sinal/sintoma } \\
\text { até data de } \\
\text { notificação }\end{array}$ & $3,4 \pm 2,6$ & 3,0 & $14 / 0$ & $3,5 \pm 3,0$ & 3,0 & $14 / 0$ & $0,00 / 0,004$ & 0,298 & -- & 0,941 \\
\hline
\end{tabular}

SD: Standardabweichung; Max.: Maximal; Min.: Minimum.; Männer.: Männlich; Fem.: Weiblich.

Die Berechnung des CCS nach Altersgruppen in der Gemeinde Macapá ergab, dass die Altersgruppe von 30-39 Jahren den höchsten SCC darstellte, der 38,99 Verdachtsfälle pro 100.000 Einwohner dieser Altersgruppe betrug. Unabhängig vom Alter wurden 21,26 Fälle pro 100.000 Personen gemeldet (Tabelle 1).

Die häufigsten Anzeichen und Symptome unter den analysierten Verdachtsfällen waren Husten, Fieber, laufende Nase, Halsschmerzen, Kopfschmerzen, Atembeschwerden, Admya, Dyspnoe/Aquipnoe und Myalgie. So hatten $82,2 \%$ der Patienten Husten, 68,2\% Fieber, 58,9\% laufende Nase, Halsschmerzen (57,0\%), Kopfschmerzen (56,1\%), Atembeschwerden (53,3\%), Adidämie $(36,4 \%)$, Dyspnoe/Aquipnoe (30,8\%) und Myalgie (27,1\%). Der Unterschied in Häufigkeit und Prozentsatz zwischen den Geschlechtern kann in Schaubild A und Schaubild B (Abbildung 1) beobachtet werden.

RC: 71076

Verfügbar in: https://www.nucleodoconhecimento.com.br/gesundheit/von-covid-19-inmacapa 
Abbildung 1 Häufigkeit und Prozentsatz der Anzeichen und Symptome, die durch Verdachtsfälle von COVID-19 dargestellt werden, analysiert, nach Geschlecht und Allgemein. Macapa, 13.-21. März 2020.
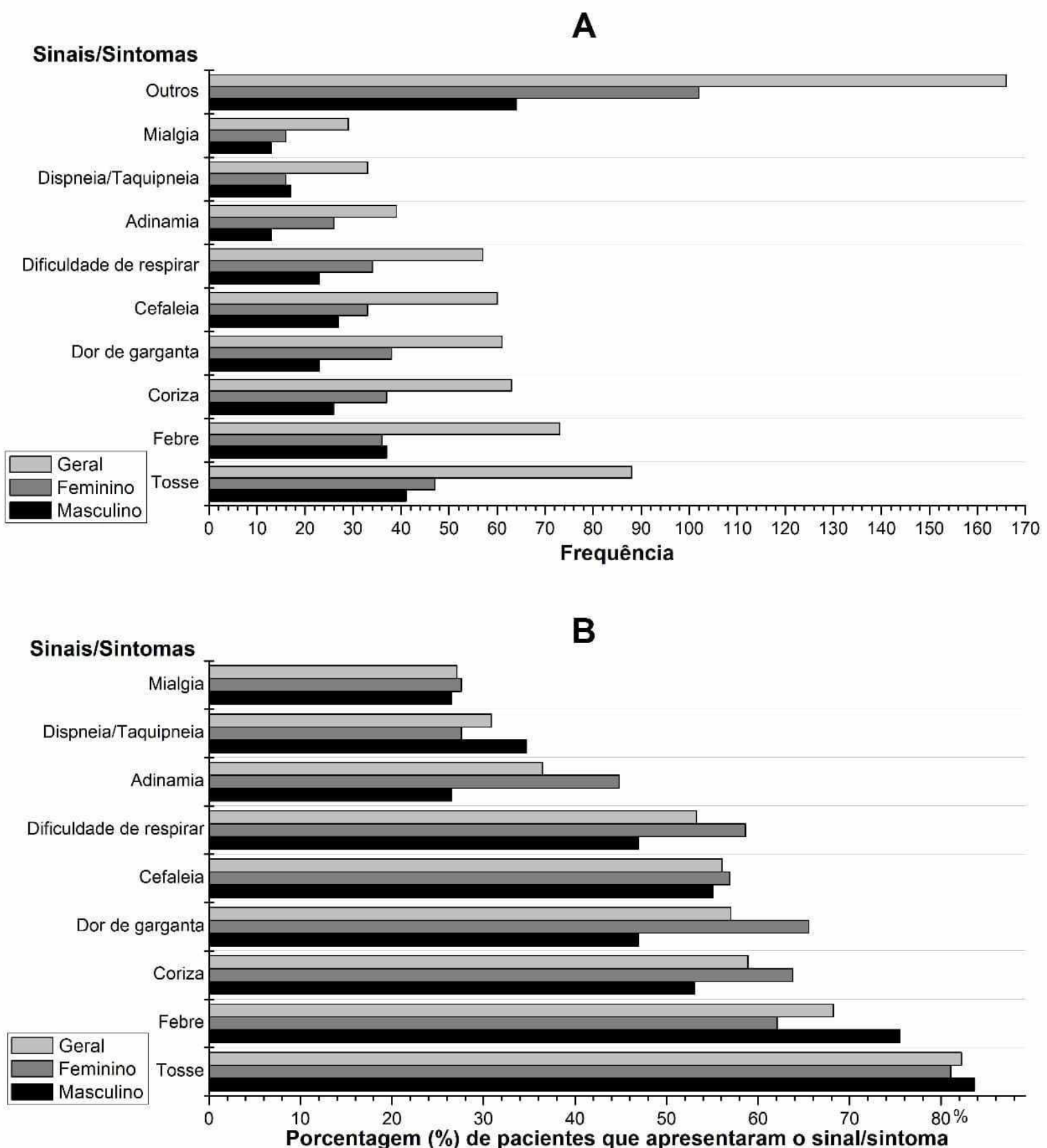

Die durchschnittliche Anzahl der Anzeichen und Symptome betrug pro analysierten

$\mathrm{RC}: 71076$

Verfügbar in: https://www.nucleodoconhecimento.com.br/gesundheit/von-covid-19-inmacapa 
Verdachtsfall $\bar{x}=6,3 \pm 3,5$. Zwischen den Geschlechtern gab es keinen statistisch signifikanten Unterschied zwischen den dargestellten quantitativen Anzeichen/Symptomen $(U=1,311 ; p=0,488)$. Die Zeit in Tagen, zwischen dem Datum des ersten Zeichens und dem Symptom und dem Datum der Meldung des Verdachtsfalls war $\bar{x}=3.4 \pm 2.8$, mit einem Median von 3 Tagen. In diesem Zeitintervall gab es keinen signifikanten Unterschied zwischen den Geschlechtern $(U=1.221,5$; $\mathrm{p}=0,941$ ) (Tabelle 3).

Nur 30 (28,04\%) Personen, unter den analysierten Verdachtsfällen, präsentierten frühere Morbiditäten, von den 107 Fällen, die in diesem Ite analysiert wurden. Die häufigste Morbidität war "Herz-Kreislauf-Erkrankungen, einschließlich Bluthochdruck" $(n=13 ; 43,33 \%)$, gefolgt von "chronischen Lungenerkrankungen" $(n=6 ; 20,00 \%)$ und "Diabetes" ( $n=5 ; 16,66 \%)$ (Tabelle 2).

Die häufigste Beschäftigung war "Gesundheitsfachkraft" ( $n=9 ; 8,57 \%)$ und 88 (83,81 \%) Fälle, von den 105 in diesem Itact analysierten, wurden als "andere" bezeichnet. Etwa 81,5\% der Fälle wurden von Gesundheitsbehörden gemeldet.

Von den 108 Verdachtsfällen waren 18 Personen (16,16\%) 16 (14.81\%) Kontakt mit verdachtigen oder bestätigten Fällen von COVID-19 und nur 7 (6,5\%) diese beiden Bedingungen wurden aufgedeckt. Diese beiden Bedingungen wurden nur in drei der 108 Fälle ignoriert.

\section{ANALYSE DER INCIDENCE DER KONFIRMATEN VON COVID-19 IN MACAPUND ANDEREN KAPITALEN}

Die Inzidenz von bestätigten Fällen von COVID-19 in Macapá, bis zum 26. März 2020, einen Monat nach dem ersten bestätigten Fall in Brasilien, betrug 0,397 Fälle pro 100.000 Einwohner. Die geringste Inzidenz unter den brasilianischen Hauptstädten hatte Macapá. Die mittlere Inzidenz in den nationalen Hauptstädten $(n=27)$ betrug $\bar{x}=2,94 \pm 2,19$ und median von 2,31. Die Hauptstadt mit der höchsten

RC: 71076

Verfügbar in: https://www.nucleodoconhecimento.com.br/gesundheit/von-covid-19-inmacapa 
Inzidenz war Fortaleza, die Hauptstadt von Ceará, mit 8,32 Fällen pro 100.000 Einwohner (Abbildung 2).

Abbildung 2 Boxplot-Diagramm, das die Inzidenz von COVID-19 in brasilianischen Hauptstädten darstellt, nach untersuchten Gruppen. Brasilianische Hauptstädte, Brasilien, vom 26. Februar 2020 bis 26. März 2020. ("outlier", repräsentiert die Hauptstadt Rio Branco, Acre).

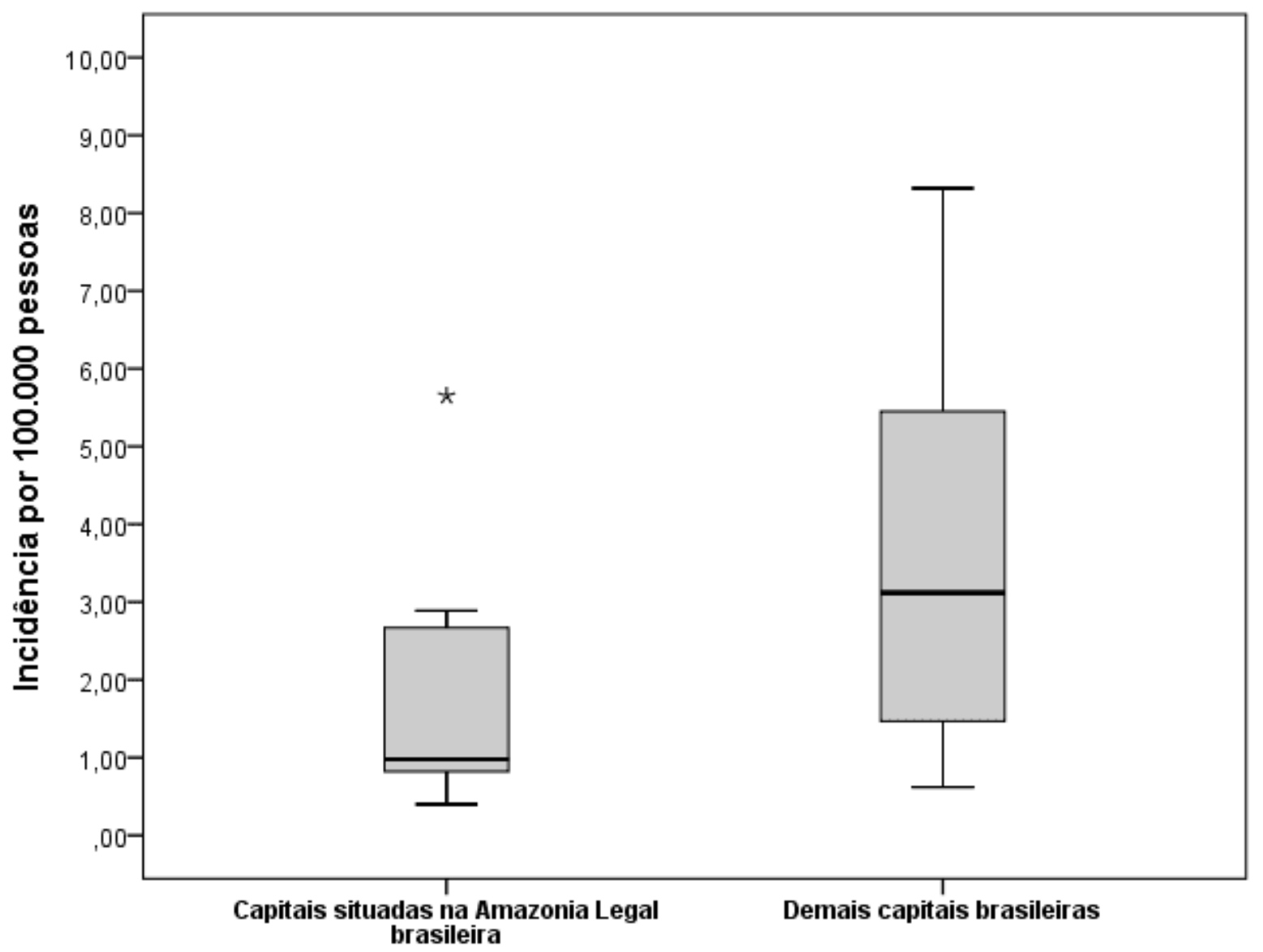

Die durchschnittliche Inzidenz von Hauptstädten im brasilianischen legalen Amazonas lag $\bar{x}=1,86 \pm 1,70$ und median von 0,98 . Die Hauptstadt in dieser Region mit der höchsten Inzidenz war Rio Branco, die Hauptstadt von Acre, mit 5,65 Fällen pro 100.000 Einwohner (Abbildung 2). Es gab einen signifikanten Unterschied

RC: 71076

Verfügbar in: https://www.nucleodoconhecimento.com.br/gesundheit/von-covid-19-inmacapa 
zwischen den Inzidenzen von Hauptstädten im Legal Amazon ( $n=9 ; \bar{x}=1,86 \pm 1,70$; Median $=0,98)$ und den Inzidenzen anderer nationaler Hauptstädte $(n=18$; $\bar{x}=3,48 \pm 2,24$; Median $=3,12)(U=41,5 ; p=0,041)$.

Die Inzidenzen in den Hauptstädten zeigten eine positive und signifikante Korrelation mit der demografischen Dichte (DD) der Hauptstädte $(r=0,416 ; p=0,031)$, der Anzahl der Gesundheitseinrichtungen pro Kapital je Kapital $(r=0,539 ; p=0,004)$ und mit der HDI der Hauptstädte $(r=0,436 ; p=0,023)$. Und negative und signifikante Korrelation mit dem prozentualen Anteil armer Menschen nach Kapital $(r=-0.409 ; \mathrm{p}=0.034)$ (Abbildung 3).

RC: 71076

Verfügbar in: https://www.nucleodoconhecimento.com.br/gesundheit/von-covid-19-inmacapa 
Abbildung 3 Abbildung Graphiken der Korrelation von Pearson zwischen Inzidenz und: demografischer Dichte; die Zahl der Gesundheitseinrichtungen, den Human Development Index und den Prozentsatz der armen Menschen. (MCP: Macapá; $r$ : Pearson-Korrelationskoeffizient).
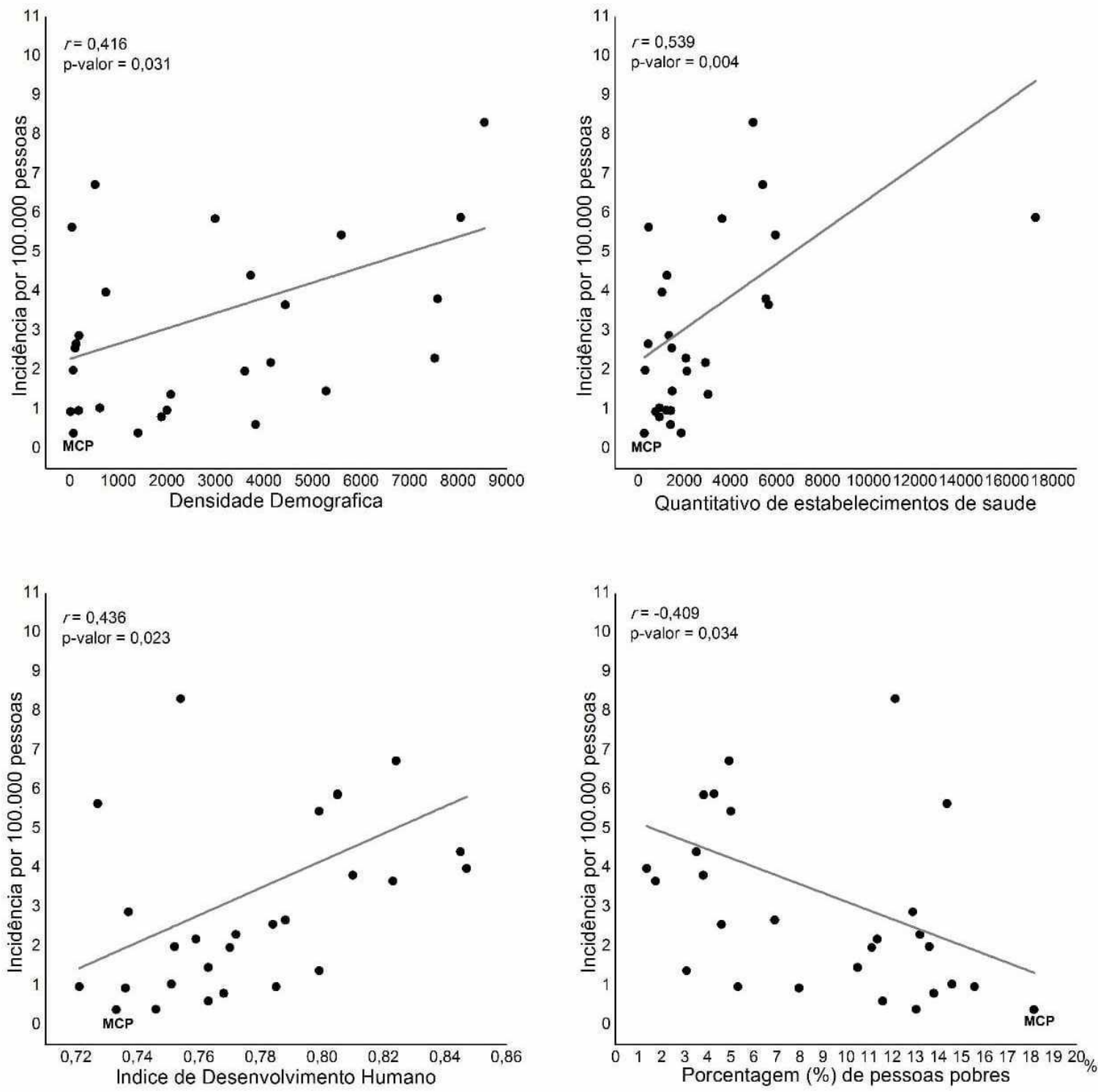

RC: 71076

Verfügbar in: https://www.nucleodoconhecimento.com.br/gesundheit/von-covid-19-inmacapa 


\section{DISKUSSION}

Der Anteil der bestätigten Fälle ( $n=3 ; 2,78 \%$ ) unter den insgesamt 108 als Verdächtige gemeldeten Personen in der Gemeinde Macapá war höher als in anderen Staaten wie Mato Grosso und Minas Gerais, die bis zum 20. März 20201 $(0,81 \%)$ bestätigt hatten. 123 gemeldete und 38 (0,92\%) Fälle von 4.122 (SESAMT, 2020; SESAMG, 2020a). Das epidemiologische Profil der drei in der vorliegenden Studie analysierten Fälle mit COVID-19 ähnelt dem Profil der ersten vier bestätigten Fälle in Minas Gerais, was die Vorherrschaft von Frauen und die Geschichte der Auslandsreise in allen Fällen betrifft, aber es gibt einen Unterschied von etwa 10,5 Jahren im Durchschnittsalter (SESAMG, 2020b). Die durchschnittliche Anzahl der Tage zwischen dem ersten Zeichen und dem Symptom und dem Datum der Meldung der drei bestätigten Fälle lag 0,4 Tage unter dem allgemeinen Durchschnitt. Dies verkürzt die Zeit bis zur Diagnose und Behandlung. es reduziert auch die Zeit, die der infizierte Patient in Kontakt mit anderen Menschen mit der Möglichkeit des Kontakts und der Ansteckung bleibt (WHO, 2020a; ZHANG et al., 2020).

Die Dominanz der Frauen ( $n=59 ; 54,62 \%)$, von insgesamt 108 Verdachtsfällen in Macapa, analysiert in dieser Studie, bestätigt das Profil der von SESAMG (2020c) am 3. März 2020 (56,0\% weiblich) offenbarten Verdachtsfälle mit dem Profil der Verdachtsfälle, die von SESAMA (2020) am 19. März 2020 (62,1\% weiblich) offengelegt wurden, und unterscheidet sich wenig vom Profil der Verdachtsfälle in Brasilien. , veröffentlicht vom Gesundheitsministerium (MS) am 10. Februar 2020 (53\% männlich) (BRASIL, 2020b). Diese Dominanz kann durch die geringe Mehrheit der Frauen im Verhältnis zu den Männern in Macapá (BRASIL, 2018b) und durch Frauen als wichtigste öffentliche Gesundheitsdienste in Brasilien (LEVORATO et al., 2014).

Das mittlere allgemeine Alter der Verdächtigen in der vorliegenden Studie, $\bar{x}$ =34,2 $\pm 14,9$ (Median=34 Jahre), liegt nahe am Profil in Minas Gerais, 33 Jahre alt (SESAMG, 2020c). Bis zum 19. März 2020 waren im Bundesstaat Ceará 42,7 \% der

RC: 71076

Verfügbar in: https://www.nucleodoconhecimento.com.br/gesundheit/von-covid-19-inmacapa 
Verdächtigen Frauen im Alter zwischen 20 und 49 Jahren (SESACE, 2020). Die Ähnlichkeit zwischen dem Mittleren Alter der Geschlechter wurde erwartet, unter Verwendung der Studie von ZHANG et al., (2020), die einen Frauenanteil von 0,99 Männern/1,0 und eine Dominanz von 89,8 \% in der Altersgruppe der 30- bis 79Jährigen in Wuhan, China, beschrieb.

Die Altersgruppe, unabhängig vom Geschlecht, mit der höchsten Anzahl von Verdachtsfällen, die in der vorliegenden Studie berichtet wurde, betrug 30-39 Jahre, mit 27,1 \% der Fälle, sehr nahe am Prozentsatz von 20-29 (25,2\%). Ähnliche Prozentsätze mit gleichem Gleichgewicht für Verdachtsfälle wurden auch im Epidemiologischen Bericht vom 19. März 2020 im Bundesstaat Rio Grande do Sul (SESARS, 2020) beschrieben. Diese Ähnlichkeit zwischen dem einfachen Prozentsatz der Altersgruppen der Verdachtsfälle erklärt sich aus der prozentualen Verteilung der Bevölkerung nach Altersgruppen von Macapá (20-29=18,2\%; 30-39= $14,5 \%)$. und der Staat Rio Grande do Sul (20-29=14,0\%; 30-39=14,6\%) (BRASIL, 2018a). Und auch, weil Menschen im Alter zwischen 26 und 49 Jahren, in denen die beiden Altersgruppen 20-29 und 30-39 enthalten sind, diejenigen sind, die am meisten Gesundheitsdienste in Brasilien suchen (LEVORATO et al., 2014).

Bei der Analyse des CCS dieser beiden Altersgruppen 38,99 (30-39 Jahre) und 29,77 (20-29 Jahre) Fälle pro 100.000 Personen, berechnet in dieser Studie, ist es möglich, zu bemerken, dass die Zahl der Meldungen pro 100.000 Personen in der Altersgruppe der 30-39-Jährigen um 31,0\% höher ist als in 20-29 Jahren. Diese Dominanz des 30-39-Jahres-Intervalls lässt sich durch diese Altersgruppe erklären, die in Brasilien am häufigsten Gesundheitsdienste anstrebt (26-49 Jahre), sowie durch den möglichen Zusammenhang zwischen der Nachfrage nach Gesundheitsdienstleistungen und der wirtschaftlichen Produktivität der Arbeit. Im Bundesstaat Amapá ist die produktivste Altersgruppe mit der höchsten Zahl von Arbeitnehmern (formale Beschäftigung) 41.365 (31,3 \%) 30-39 Jahre alt (BRASIL, 2018b; LEVORATO et al., 2014). Die Nachfrage nach und der Zugang zur

$\mathrm{RC}: 71076$

Verfügbar in: https://www.nucleodoconhecimento.com.br/gesundheit/von-covid-19-inmacapa 
Gesundheit wird auch durch das Einkommen beeinflusst. Menschen mit einer besseren wirtschaftlichen Lage suchen mehr und haben einen besseren Zugang zu Gesundheitsdienstleistungen. In Brasilien ist die wirtschaftliche Lage der über 30 Jährigen im Vergleich zu den unteren Altersgruppen wesentlich besser (BRASIL, 2018c; TRAVASSOS et al., 2006).

Die in der vorliegenden Studie untersuchten vorherrschenden Anzeichen und Symptome, Husten (82,2\%); Fieber (68,2\%); Halsschmerzen (57,0\%); Dyspnoe/Aquipnoe (30,8\%), wurden als die häufigsten unspezifischen Anzeichen und Symptome in bestätigten Fällen von COVID-19 (BRASIL, 2020a; LI et al., 2020; WHO, 2020a). Es gab auch Übereinstimmung mit den Anzeichen und Symptomen von Verdachtspatienten, die von SESARS (2020) und BRASIL (2020b) beschrieben wurden. Die größte Diskrepanz wurde bei "Sore Throat" beobachtet, die 57,0\% der Fälle in der aktuellen Studie und verglichen mit 13,9\% von 55.924 bestätigten chinesischen Fällen (WHO, 2020a) aufzeigte. Dieser Unterschied kann darauf zurückzuführen sein, dass nur $2,78 \%$ der in dieser Studie analysierten Fälle bestätigt werden, während alle von der WHO (2020a) beschriebenen Fälle eine bestätigte Diagnose von COVID-19 haben. Eine andere Möglichkeit wäre die wahrscheinliche Koinfektion durch Bakterien in einigen Fällen (ZHANG et al., 2020).

In der vorliegenden Studie lag die mittlere Zeit in Tagen zwischen dem Datum des ersten Anzeichens/Symptoms, das vom Patienten gemeldet wurde, und dem Datum der Meldung des Verdachtsfalls $\bar{x}=3,4 \pm 2,8$. In $50 \%$ der als vermutet gemeldeten Fälle betrug die Verzögerung bis zur Meldung mehr als drei Tage (Median=3). Dies deutet darauf hin, dass Patienten, die als COVID-19 vermutet wurden, symptomatisch blieben, im Durchschnitt 3,4 Tage, mit möglichem Kontakt mit anderen Menschen. Nach Angaben der WHO (2020a) erfolgt die Übertragung der überwiegenden Mehrheit der Fälle durch symptomatische Patienten, obwohl sie selten von einem asymptomatischen Patienten auftreten kann, wie ROTHE et al. (2020).

$\mathrm{RC}: 71076$

Verfügbar in: https://www.nucleodoconhecimento.com.br/gesundheit/von-covid-19-inmacapa 
Die früheren Morbiditäten, die von den Verdachtsfällen der vorliegenden Studie dargestellt wurden, ähneln denen, die bei 20.812 chinesischen Patienten beschrieben wurden, die mit COVID-19 in der Studie von ZHANG et al. bestätigt wurden. (2020), die Diabetes (5,3\%), Bluthochdruck (12,8\%), andere Herz-KreislaufErkrankungen (4,2\%) und chronische Lungenerkrankungen (2,4\%) Morbiditäten, die mehr mit COVID-19 in Verbindung gebracht werden, wobei Bluthochdruck und andere Herz-Kreislauf-Erkrankungen eher mit ungünstigen Ergebnissen zusammenhängen.

Die einzige in dieser Studie erwähnte Berufsgruppe war die Gesundheit ( $n=9 ; 8,57$ $\%$ ), die eine der wichtigsten Risikogruppen für COVID-19 ist (GALLASCH et al., 2020; ZHANG et al., 2020). Darüber hinaus gab es eine hohe Anzahl von Markierungen als "sonstige" ( $n=88 ; 83,82 \%)$. Dies kann die Beurteilung anderer möglicher Risikoberufe beeinträchtigen, die mit einem höheren Infektionsrisiko zusammenhängen können ( $\mathrm{KOH}, 2020$; ZHANG et al., 2020;) Die überwiegende Mehrheit der in dieser Studie analysierten Verdachtsfälle wurde vom Einheitliches Gesundheitssystem (SUS) gemeldet, das die Bedeutung und Souveränität dieses staatlichen Systems demonstrieren kann, das der überwiegenden Mehrheit der brasilianischen Bevölkerung auf integrale und freie Weise dient (BRASIL, 2020f; VIANA et al., 2009).

Eine signifikante Korrelation der Inzidenz von COVID-19 wurde mit bestimmten Indikatoren identifiziert. Zumindest in der Anfangsphase, bis zum 26. März 2020, der Verbreitung des Virus, besteht ein Zusammenhang zwischen höheren Inzidenzwerten von COVID-19 und der Bevölkerung, die in Städten mit guten Indikatoren für die soziale Entwicklung lebt.

Unter der Korrelation, die mit positiver Assoziation analysiert wird, wird hervorgehoben, dass mit demografischer Dichte (DD) durchgeführt. Die meisten Fälle von COVID-19/100.000 Menschen korrelieren mit einer größeren Anzahl von Menschen, die das gleiche Gebiet an einem bestimmten Ort besetzen. Es ist wichtig

RC: 71076

Verfügbar in: https://www.nucleodoconhecimento.com.br/gesundheit/von-covid-19-inmacapa 
zu betonen, dass die Agglomeration von Individuen die Ausbreitung des Virus erleichtert, da Infektionen in Familiengruppen sowie in Gesundheitsfachleuten das Auftreten von Mensch-Zu-Mensch-Übertragung bestätigen, hauptsächlich durch engen Kontakt (READ et al., 2020; BRASIL, 2020i). In China waren die am stärksten besetzten Städte mit den meisten täglichen Flügen betroffen (LAl et al., 2020; READ et al., 2020). Die in dieser Studie bis Februar 2020 mit der geringsten Inzidenz hervorgehobene Stadt Macapa hatte bis Februar 2020 nur Verbindungen zu täglichen und direkten kommerziellen Flügen nach Brasília, Bundesland, und Belém, Pará (BRASIL, 2020j).

Die Bevölkerungsmerkmale im Zusammenhang mit COVID-19 werden noch wenig diskutiert. Einige Studien, wie die von der WHO (2020a), können das Ergebnis dieser Assoziation rechtfertigen, da sie eine höhere Übersendefähigkeit in Regionen mit höherer Bevölkerungskonzentration zeigen. Darüber hinaus schlägt das Gesundheitsministerium generell vor, dass bestimmte Ausbrüche in Gebieten mit hoher Bevölkerungsdichte eine höhere Verbreitungswahrscheinlichkeit haben und somit Ereignisse darstellen, die große Auswirkungen auf die öffentliche Gesundheit haben können (BRASIL, 2009).

Eine weitere räumliche Kartierungs- und Analysestudie, die in China durchgeführt wurde, zeigte, dass die Verteilung der COVID-19-Fälle nicht zufällig war. Die Konzentrationspunkte der Fälle zu Beginn der Epidemie beschränkten sich auf Gebiete mit größerer wirtschaftlicher Entwicklung und Bevölkerungsdichte. Diese Faktoren hängen mit der größeren Zahl von Fällen zusammen, da sie eine größere Pendelmobilität, eine größere Anzahl von Fahrten und eine größere Anzahl von Gesundheitseinrichtungen bieten, die für die Durchführung von Pflege- und Diagnoseeinrichtungen qualifiziert sind (FAN et al., 2020a; LAl et al., 2020; OLIVEIRA et al., 2019; ZASLAVSKY et al., 2017).

Diese Beweise bestätigen und rechtfertigen die Ergebnisse der vorliegenden Studie, da bestimmte soziodemographische und Gesundheitsindikatoren eine positive und

RC: 71076

Verfügbar in: https://www.nucleodoconhecimento.com.br/gesundheit/von-covid-19-inmacapa 
signifikante Korrelation mit der Inzidenzrate von COVID-19 unter den Hauptstädten zeigten, d. h. die Quantifizierung und Analyse der zwischen dem 26. Februar und dem 26. März in Brasilien registrierten Fälle zeigten, dass die Hauptstädte mit höherer DD, höherer Human Development Index (HDI) und mehr Gesundheitseinrichtungen höhere Inzidenzraten aufwiesen.

Gute soziale Indikatoren können mit einer höheren Anzahl von schwimmenden Populationen zusammenhängen, d. h. mit der, die zu einem bestimmten Zeitpunkt, für einen kurzen Zeitraum und auf der Suche nach verschiedenen Aktivitäten im Hoheitsgebiet vorhanden ist (BRASIL, 2011). So können Städte mit guten sozialen Indikatoren, die Möglichkeiten des Tourismus und der Wirtschaft bieten, eine höhere Inzidenz von Infektionen darstellen, vor allem solche, die von Mensch zu Mensch übertragen werden, weil sie dieser saisonalen gebietsfremden Bevölkerung mehr Anziehungskraft bieten, da die Person, die diese Gruppe zusammensetzt, ein potenzieller Träger für die Übertragung des Virus ist (FAN et al., 2020b). Eine Korrelationsstudie von FAN et al. (2020b), bewertete den Zusammenhang zwischen der quantitativen schwimmenden Population von Wuhan und der Anzahl der bestätigten Fälle von COVID-19, die als Ergebnis einen Korrelationskoeffizienten von 0,84 erhalten. Dies deutet darauf hin, dass in dieser Region mehr bestätigte Fälle auftreten, wenn eine Region eine größere Anzahl gebietsfremder Personen hat.

Die negative Korrelation ( $r=-0.409 ; \mathrm{p}=0.034)$ zwischen der Inzidenz von COVID-19 und dem Prozentsatz der armen Menschen pro Kapital zeigte, dass je höher die Zahl der Fälle/100.000 Menschen, desto geringer der Prozentsatz der armen Menschen, die in der Hauptstadt leben. Diese Feststellung bestätigt die Existenz des Zusammenhangs zwischen Inzidenz und HDI, der auch in der vorliegenden Studie beobachtet wurde.

Da zunächst eine größere Zahl von exportierten infizierten Fällen die Möglichkeit einer höheren Zahl von Sekundärfällen in der Gemeinschaft unmittelbar beeinflusst, können Hauptstädte mit dem höchsten Anteil armer Menschen und folglich weniger

RC: 71076

Verfügbar in: https://www.nucleodoconhecimento.com.br/gesundheit/von-covid-19-inmacapa 
Reisenden zunächst geringere Inzidenzen haben (KUCHARSKI et al., 2020; ROSA, 2006). Im Allgemeinen hat diese Bevölkerung weniger Zugang zu Bildungs-, Informations- und Gesundheitsressourcen. Diese Faktoren können die Erkennung von Anfangszeichen/Symptomen behindern und neben der Beschränkung des Zugangs zu Gesundheitseinrichtungen auch zu Kovid-19-Identifikationsfehlern führen und folglich zu einer Untererfassung von Diagnosen führen (CAMPELLO et al., 2018).

In Übereinstimmung mit diesen Beobachtungen zeigten die Ergebnisse der vorliegenden Studie, dass Macapa die Hauptstadt mit der niedrigsten Inzidenz von COVID-19 pro 100.000 Menschen ist, nachdem ein Monat nach der Bestätigung des ersten Falls in Brasilien. Und das hat in diesem Einklang eine der niedrigsten IDHs und eine der niedrigsten DDs unter den Hauptstädten, die kleinste Anzahl von Gesundheitseinrichtungen im Land unter den Hauptstädten, eine begrenzte Anzahl von täglichen kommerziellen Flügen und die höchste prozentuale Beteiligung armer Menschen unter allen nationalen Hauptstädten (ATLAS BRASIL, 2020; BRASIL, 2020g; BRASIL, 2020j).

Die Inzidenz bis zum 26. März 2020 in der Gruppe der Hauptstädte der brasilianischen legalen Amazonasregion $(n=9 ; \bar{x}=1,86 \pm 1,70$; Median $=0,98)$ war deutlich niedriger $(U=41,5 ; p=0,041)$ als die Gruppe anderer brasilianischer Hauptstädte ( $\mathrm{n}=18 ; \bar{x}=3,48 \pm 2,24$; Median $=3,12)$. Dieser Unterschied kann auf die sozio-räumliche Isolation des Legal Amazon ascin al, in Bezug auf den Rest von Brasilien, von VIANA et al beschrieben. (2009). Ein Beispiel für diese Isolation ist die Hauptstadt Macapá, in der 59,5 \% der Bevölkerung von Amapa leben, die nicht landfest mit dem Rest des Landes verbunden ist (BRASIL, 2020f; DRUMMOND, 2000) und stellte in dieser Studie die niedrigste Inzidenz $(0,397)$ von COVID-19 unter den brasilianischen Hauptstädten vor.

Dieser Unterschied in der Inzidenz von COVID-19 kann neben der sozio-räumlichen Isolation mit klimatologischen und städtischen Faktoren im Amazonasgebiet

RC: 71076

Verfügbar in: https://www.nucleodoconhecimento.com.br/gesundheit/von-covid-19-inmacapa 
zusammenhängen, wie hohe relative Luftfeuchtigkeit und demografische Dichte (BRASIL 2020f; SILVA et al., 2013; WANG et al., 2020).

Zunächst gab es in den großen urbanen Zentren des brasilianischen Amazonasgebiets eine Geschichte von Epidemien von Viruskrankheiten, die durch Mücken wie Zika und Gelbfieber übertragen werden. Die klimatischen Faktoren des Amazonas-Ökosystems, die dazu beitragen, die endemische Übertragung und/oder das Auftreten von epidemischen Wellen aufrechtzuerhalten, indem sie die Vermehrung von Mücken fördern (FARIA et al., 2018; GIOVANETTI et al., 2020), können die Übertragung jedoch benachteiligen von COVID-19 im Amazonas.

In-vitro-Studien von SILVA et al. (2013), von WANG et al. (2020) und KAMPF et al., (2020) hängt eine hohe relative Luftfeuchtigkeit konsequent mit der geringeren Lebensfähigkeit von umhüllten Viren zusammen, die Atemwegserkrankungen wie Coronavirus verursachen. Dies kann auf eine geringere Virusstabilität bei höheren Temperaturen zurückzuführen sein und Atemtröpfchen bleiben weniger Zeit in der Luft mit hoher Luftfeuchtigkeit (WANG et al., 2020). Die Ergebnisse von OLIVEIROS et al. (2020) zeigte, dass die Zahl der Fälle von COVID-19 in einem linearen Regressionsmodell aufgrund dieser klimatischen Faktoren nur 18,0 \% des Einflusses erlitt. Während Bevölkerungsdichte, städtischer Verkehr, kulturelle Aspekte, öffentliche Gesundheitspolitik und Beseitigungsmaßnahmen 82,0 \% beeinflussen.

Dies zeigt, dass möglicherweise nur klimatische Faktoren nicht ausreichen werden, um die Zunahme der Zahl der Fälle einzudämmen, und andere Maßnahmen sind notwendig, wie die soziale Entfernung, die von der Staatsregierung in einigen föderalen Einheiten des brasilianischen legalen Amazonas verfügt wurde, wie Amapá (AP, 2020; RIPAP, 2020). In Ermangelung wirksamer Impfstoffe oder antiviraler Medikamente, Maßnahmen wie der sozialen Entzug, hat es das Potenzial, die Exzentrizität der COVID-19-Inzidenzkurve zu verringern, seinen Höhepunkt abzuflachen und die akute Überlastung des Gesundheitssystems zu verringern. Es ist von wesentlicher Bedeutung, dass die Behörden über epidemiologische

RC: 71076

Verfügbar in: https://www.nucleodoconhecimento.com.br/gesundheit/von-covid-19-inmacapa 
Warnungen und die Beiträge der wissenschaftlichen Gemeinschaft informiert sind (HAFFAJEE et al., 2020; PREM et al., 2020; RIPAP, 2020; WU et al., 2020).

\section{FAZIT}

Das in dieser Studie beschriebene Profil der Verdachtsfälle ähnelt dem Profil von Verdachtsfällen und in einigen Punkten sogar des Profils bestätigter Fälle in der wissenschaftlichen Literatur, obwohl die Zahl der Studien über das Profil von Verdachtsfällen derzeit begrenzt ist. Orte wie Macapá, die besondere soziale, geografische und wirtschaftliche Merkmale aufweisen, können ein Muster der Verbreitung von COVID-19 aufdecken, was es interessant macht, das epidemiologische Profil der Fälle kontinuierlich und streng zu überwachen.

Im Vergleich zu anderen brasilianischen Ortschaften hat Macapa einen besseren Prozentsatz als die Optionen zur Bekämpfung des Virus. Die Koordinierung der Fallbenachrichtigung kann jedoch durch eine effektive Kommunikation zwischen verschiedenen Interessenträgern, einschließlich Gesundheitseinrichtungen und berufsprofessionalen Gesundheitsgesellschaften, optimiert werden.

Die Rolle der Regierung bei der Sensibilisierung der Öffentlichkeit für COVID19 ist entscheidend für die Verringerung der Krankheitslast. Darüber hinaus kann die Registrierung der Besetzung des gemeldeten Falles verbessert werden, einschließlich z. B. Von Motoboys, Post- und öffentlichen Sicherheitsservern, die wahrscheinlich mehr expositionserfahren sind als andere Berufstätige.

Mehr als 80,0 \% der analysierten Fälle wurden von Gesundheitsbehörden gemeldet. Dies zeigt die Bedeutung eines robusten staatlichen Gesundheitssystems, das einen Unterschied bei der Eindämmung der COVID-19-Epidemie sowohl in Macapa als auch im brasilianischen Amazonasgebiet und im Rest des Landes machen kann.

Die Korrelation zwischen der Inzidenz von COVID-19 und den sozialen, soziodemografischen und gesundheitlichen Indikatoren lässt schließen, dass die

$\mathrm{RC}: 71076$

Verfügbar in: https://www.nucleodoconhecimento.com.br/gesundheit/von-covid-19-inmacapa 
Hauptstädte mit der größten wirtschaftlichen, bevölkerungs- und gesundheitlichen Entwicklung nach einem Monat des ersten Falls in Brasilien am stärksten von Coronavirus betroffen waren. Diese Erkenntnis ist vor allem auf den größeren Zustrom von Reisenden zurückzuführen. Daher können restriktive Maßnahmen der Mobilität der Bevölkerung sowie des Sozialurlaubs wirksamer sein, wenn sie in Hauptstädte mit einem großen Personenstrom gelenkt werden.

Die besonderen Merkmale von Macapá, wie in dieser Studie hervorgehoben, verlangsamten zunächst die Übertragbarkeit des Coronavirus in der Hauptstadt von Amapá. Die Tatsache, dass Macapá weit von der wichtigsten nationalen sozioökonomischen Achse entfernt ist, keine Landrouten der Kommunikation mit anderen Großstädten hat und eine geringere Anzahl von Flügen in andere Städte hat, scheint bis zum 26. März 2020 zu der geringen Inzidenzrate in diesem Ort beigetragen zu haben. Macapá ist die brasilianische Hauptstadt mit der geringsten Anzahl an Gesundheitseinrichtungen. Dies wirft sicherlich Fragen über asymptomatische Übertragungen auf, da die öffentliche Gesundheitsinfrastruktur in dieser Stadt mit einigen Einschränkungen konfrontiert ist. Der prozentuale Anteil der in dieser Studie beschriebenen Diagnosen für Macapá ist jedoch nach wie vor höher als der einiger brasilianischer Staaten mit besseren sozioökonomischen und gesundheitlichen Indikatoren. Wahrscheinlich, weil die Gemeinden im Landesinneren über einen kleineren staatlichen Gesundheits- und Verwaltungsapparat verfügen. Ein größerer Staatsapparat könnte in der Lage sein, Verhaltensweisen und Protokolle in Gesundheitseinheiten direkt zu beeinflussen, was die Wirksamkeit diagnostischer Verhaltensweisen erhöhen kann. Die Ausbildung von Gesundheitsfachkräften, die in vielen dieser Gemeinden tätig sind, kann auch die Wirksamkeit der Diagnose beeinflussen.

Geographische Faktoren sind möglicherweise Influencer für eine geringere Ausbreitung des Virus im Amazonasgebiet, vor allem in kleinen und mittleren städtischen Zentren, wie Macapá. Diese Faktoren sind zusammen mit staatlichen

RC: 71076

Verfügbar in: https://www.nucleodoconhecimento.com.br/gesundheit/von-covid-19-inmacapa 
Maßnahmen zur sozialen Verdeintung der Bevölkerung, Investitionen in Prävention und Sensibilisierung sowie in wissenschaftliche Forschung/Entwicklung zur Bekämpfung von COVID-19 von wesentlicher Bedeutung, um die akuten Auswirkungen auf das öffentliche Gesundheitssystem zu verringern, das in den meisten dieser städtischen Zentren aufgrund der historischen Ungleichheit zwischen den brasilianischen Regionen für diese Art von Epidemie fragil ist.

\section{DANKE}

Wir danken João Silvestre da Silva-Júnior, Arzt in Gesundheitswesen von der Fakultät für Gesundheitswesen der Universität São Paulo, und Virgílio Amaral da Cunha Junior, einem Journalisten mit Abschluss an der Bundesuniversität Viçosa, für die Zusammenarbeit.

\section{REFERENZEN}

AP. Governo do Estado do Amapá. Decreto n 1.414 de 19 de Março de 2020. Dispõe sobre medidas de restrição de aglomeração de pessoas com a finalidade de reduzir os riscos de transmissão do novo Coronavírus (COVID-19) e adota outras providências. Macapá, AP: 2020.

ATLAS BRASIL. Atlas do Desenvolvimento Humano no Brasil. Disponível em: < http://atlasbrasil.org.br/2013/pt/consulta/ >. Acesso em: 24 março 2020.

BRASIL. Ministério da Saúde. Secretaria de Atenção Primária à Saúde. Protocolo de Manejo Clínico do Coronavírus (Covid-19) na Atenção Primária à Saúde. 1. ed. Brasília, DF: Ministério da Saúde - Secretaria de Atenção Primária à Saúde. $2020 a$.

- Ministério da Saúde. Secretaria de Vigilância em Saúde. Boletim Epidemiológico 02: Infecção humana pelo Novo Coronavírus (2019-nCov).

RC: 71076

Verfügbar in: https://www.nucleodoconhecimento.com.br/gesundheit/von-covid-19-inmacapa 
Brasilia, DF: Ministério da Saúde - Centro de Operações de Emergências em Saúde Pública (COE-nCoV): 2020b.

- Ministério da Saúde. Secretaria de Atenção Especializada à Saúde. Protocolo de Tratamento do Novo Coronavírus (2019-nCoV). 1. ed. Brasília, DF: Ministério da Saúde - Secretaria de Atenção Especializada à Saúde. 2020c.

. Ministério da Saúde. Coronavírus: 25 mortes e 1.546 casos confirmados. Brasilia, DF: 2020d. Disponível em: < https://www.saude.gov.br/noticias/agencia-saude/46573-coronavirus-25-mortes-e-1546-casos-confirmados >. Acesso em: 23 Março 2020.

Ministério da Saúde. Portaria no 454, de 20 de Março de 2020. Declara, em todo o território nacional, o estado de transmissão comunitária do Coronavírus (Covid-19). Brasília, DF: 2020e. Disponível em: < http://www.in.gov.br/en/web/dou/-/portaria-n-454-de-20-de-marco-de-2020249091587 >. Acesso em: 24 Março 2020.

Instituto Brasileiro de Geografia e Estatística. IBGE Cidades e Estados. 2020f. Disponível em: < https://www.ibge.gov.br/cidades-e-estados >. Acesso em: 23 Março 2020.

Ministério da Saúde. DATASUS. Cadastro Nacional dos Estabelecimentos de Saúde do Brasil - CNES. Quantidade por Tipo de Prestador segundo Capital. Brasília, DF: 2020g. Disponível em: < https://nam10.safelinks.protection.outlook.com/?url=http\%3A\%2F\%2Ftabnet.datasus .gov.br\%2Fcgi\%2Ftabcgi.exe\%3Fcnes\%2Fcnv\%2Festabbr.def\&amp;data=02\%7C01 \%7C\%7C5789bcef877546b7609e08d7d5ac6237\%7C84df9e7fe9f640afb435aaaaaa aaaaaa\%7C1\%7C0\%7C637212808662483071\&amp;sdata=AZP0PC\%2BzxxMGbM TJLdlJO0zLGtXCF9F3BthmFJnvqgo\%3D\&amp;reserved=0 >. Acesso em 24 Março 2020.

RC: 71076

Verfügbar in: https://www.nucleodoconhecimento.com.br/gesundheit/von-covid-19-inmacapa 
- Ministério da Saúde. Secretaria de Vigilância em Saúde. Boletim Epidemiológico 05: Doença pelo Coronavírus 2019. Brasilia, DF: Ministério da Saúde - Centro de Operações de Emergências em Saúde Pública (COE-nCoV): $2020 \mathrm{~h}$.

Ministério da Saúde. Sobre a doença. Brasília, DF: 2020i. Disponível em: < https://coronavirus.saude.gov.br/sobre-a-doenca >. Acesso em: 03 Abr. 2020.

Agência Nacional de Aviação Civil. Consulta Interativa - Indicadores do Mercado de Transporte Aéreo. Brasília, DF: 2020j. Disponível em: < https://www.anac.gov.br/assuntos/dados-e-estatisticas/mercado-de-transporteaereo/consulta-interativa/demanda-e-oferta-origem-destino >. Acesso em: 23 Mar. 2020

Instituto Brasileiro de Geografia e Estatística. Pesquisa Nacional por Amostra de Domicílios Contínua - PNAD Contínua 2016-2018, Características Gerais dos Moradores. Brasília, DF: 2018a. Disponível em: < https://www.ibge.gov.br/estatisticas/sociais/populacao/17270-pnadcontinua.html?edicao=24437\&t=downloads >. Acesso em: 24 Março 2020.

Ministério da Economia. Relação Anual de Informações Sociais: Amapá 2018. Brasília, DF: 2018b. Disponível em: < http://pdet.mte.gov.br/rais?view=default >. Acesso em: 03 Abril 2020.

Instituto Brasileiro de Geografia e Estatística. Coordenação de População e Indicadores Sociais. Síntese de Indicadores Sociais 2018: Uma Análise das Condições de Vida da População Brasileira. Brasília, DF: 2018c.

. Instituto Brasileiro de Geografia e Estatística. Amazônia Legal: Municípios da Amazônia Legal. Brasília, DF: 2014. Disponível em: < https://www.ibge.gov.br/geociencias/informacoes-ambientais/geologia/15819amazonia-legal.html?=\&t=acesso-ao-produto >. Acesso em: 24 Março 2020.

RC: 71076

Verfügbar in: https://www.nucleodoconhecimento.com.br/gesundheit/von-covid-19-inmacapa 
. Instituto Brasileiro de Geografia e Estatística - IBGE. Atlas de Saneamento

Glossário. Brasília., DF: 2011. Disponível em: https://biblioteca.ibge.gov.br/visualizacao/livros/liv53096_glossario_equipetec.pdf. Acesso em: 29 Mar. 2020.

. Ministério da Saúde. Guia de Vigilância Epidemiológica. Brasília, DF:. 2009. Disponível em: https://bvsms.saude.gov.br/bvs/publicacoes/guia_vigilancia_epidemiologica_7ed.pdf. Acesso em: 01 abr. 2020.

CAMPELLO, T.; GENTILI, P.; RODRIGUES, M.; HOEWELL, G.R. Faces da desigualdade no Brasil: um olhar sobre os que ficam para trás. Saúde em Debate. v.42 n. especial p.54-66, 2018. https://doi.org/10.1590/0103-11042018S305

CASCELLA, M.; RAJNIK, M.; CUOMO, A.; DULEBOHN, S.; DI NAPOLI, R. Features, Evaluation and Treatment Coronavirus (COVID-19). [Atualizado em 8 março 2020] Treasure Island, FL: StatPearls Publishing; 2020. Disponível em: <https://www.ncbi.nlm.nih.gov/books/NBK554776/ > Acesso em: 23 Março 2020.

DOREMALEN, N.V. et al. [Correspondece]. Aerosol and Surface Stability of SARSCoV-2 as Compared with SARS-CoV-1. Destinatário: The New England Journal of $\begin{array}{lllll}\text { Medicine. } & \text { Massachusetts, } & 17 & \text { de }\end{array}$ https://doi.org/10.1056/NEJMc2004973

DRUMMOND, J.A. Investimentos privados, impactos ambientais e qualidade de vida num empreendimento mineral amazônico: o caso da mina de manganês de Serra do Navio (Amapá). História, Ciências, Saúde-Manguinhos. Rio de Janeiro, v. 6, supl. p. 753-792, 2000. https://doi.org/10.1590/S0104-59702000000500002

FAN, J.; LIU, X.; PAN.; DOUGLAS, M.W.; BAO, S. Epidemiology of 2019 Novel Coronavirus Disease-19 in Gansu Province, China, 2020. Emerging Infectious Diseases. v. 26 n.6, 2020a. https://doi.org/10.3201/eid2606.200251

RC: 71076

Verfügbar in: https://www.nucleodoconhecimento.com.br/gesundheit/von-covid-19-inmacapa 
FAN, C. et al. Prediction of Epidemic Spread of the 2019 Novel Coronavirus Driven by Spring Festival Transportation in China: A Population-Based Study. International Jornal of Envirommental Research and Public Health. v.17 n.5, $2020 \mathrm{~b}$. https://doi.org/10.3390/ijerph17051679

FARIA, N.R. et al. Genomic and epidemiological monitoring of yellow fever virus transmission potential. Science. v.361 n. 6405 p.894-899, 2018. https://doi.org/10.1126/science.aat7115

GALLASCH, C.H.; CUNHA, M.L.; PEREIRA, L.A.S.; SILVA-JUNIOR J.S. Prevenção relacionada à exposição ocupacional do profissional de saúde no cenário de COVID19. Revista Enfermagem UERJ. v. 28 e. 49596. https://doi.org/10.12957/reuerj.2020.49596

GIOVANETTI, M. et al. Genomic and Epidemiological Surveillance of Zika Virus in $\begin{array}{lllll}\text { the Amazon Region. Cell Reports. v.30 n.7, } 2020 . & \end{array}$ https://doi.org/10.1016/j.celrep.2020.01.085

HAFFAJEE, R.L.; MELLO, M.M. Thinking Globally, Acting Locally: The U.S. Response to Covid-19. The New England Journal of Medicine, 2020. https://doi.org/10.1056/NEJMp2006740

KAMPF, G.; TODT, D.; PFAENDER, S.; STEINMANN, E. Persistence of coronaviruses on inanimate surfaces and their inactivation with biocidal agents. The Journal of Hospital Infection. v. 104, n. 3, p. 246-251, 2020. https://doi.org/10.1016/j.jhin.2020.01.022

KUCHARSKI, A.J. et al. Early dynamics of transmission and control of COVID-19: a mathematical modelling study. The Lancet: Infectous Diseases. 11 Março 2020. https://doi.org/10.1016/S1473-3099(20)30144-4

RC: 71076

Verfügbar in: https://www.nucleodoconhecimento.com.br/gesundheit/von-covid-19-inmacapa 
$\mathrm{KOH}$, D. Occupational risks for COVID-19 infection. Occupational Medicine. v.70 n.1 p. 3-5, 2020. https://doi.org/10.1093/occmed/kqaa036

LAI, S.; BOGOCH, I.I.; WALTS, E.; KHAN, K.; LI, Z.; TATEM, A. Preliminary risk analysis of 2019 novel coronavirus spread within nad beyond China. World pop. No prelo, 2020. Disponível em: < https://www.worldpop.org/resources/docs/china/WorldPop-coronavirus-spread-riskanalysis-v2.pdf >. Acesso: 02 Abril 2020.

LEVIN J.; FOX J.A.; FORDE D.R. Elementary statistics in social research. $12^{\mathrm{a}} \mathrm{ed}$. Upper Saddle River: Pearson Education; 2014.

LEVORATO, C.D.; MELLO, L.M.; SILVA, A.S.; NUNES A.A. Fatores associados à procura por serviços de saúde numa perspectiva relacional de gênero. Ciência e Saúde Coletiva. v. 19 n.4 $\quad$ p. 1263-74, 2020. https://doi.org/10.1590/141381232014194.01242013

LIPSITCH, M.; PHIL, D.; SWERDLOW, D.L.; FINELLI, L. Defining the Epidemiology of Covid-19 - Studies Needed. The New England Journal of Medicine. 2020. https://doi.org/10.1056/NEJMp2002125

LI, W. et al. Angiotensin-converting enzyme 2 is a functional receptor for the SARS coronavirus. Nature. v. 426, 2003. https://www.nature.com/articles/nature02145.pdf

LI, Y.C.; BAI W.Z.; HASHIKAWA, T. The neuroinvasive potential of SARS-CoV2 may be at least partially responsible for the respiratory failure of COVID-19 patients. Journal of Medical Virology. p. 1-4, 2020. https://doi.org/10.1002/jmv.25728

MAPA DO CORONA VÍRUS. POLATO, A.; CUNHA, R.; SORANO, V. (Coord.). Casos de coronavírus no Brasil e no Mundo: mapa e evolução. Disponível em: < https://especiais.g1.globo.com/bemestar/coronavirus/mapa-

RC: 71076

Verfügbar in: https://www.nucleodoconhecimento.com.br/gesundheit/von-covid-19-inmacapa 
coronavirus/?_ga=2.48817062.1083509909.1584787216-

1227695381.1584787197\#/.> Acesso em: 26 mar. 2020.

MCINTOSH, K. UpToDate. Coronavirus Disease 2019 (COVID-19). USA: 2020. Disponível em: < https://www.uptodate.com/contents/coronavirus-disease-2019covid-19 >. Acesso em: 23 março 2020.

NASSIRI, R. Perspective on Wuhan Viral Pneumonia. Advances in Public Health, Community and Tropical Medicine, V. 02, 2020.

OLIVEIRA, N.M.; PIFFER, M.; STRASSBURG, U. O Indicador de Desenvolvimento Regional no Território do Tocantins. Interações. v.20 n.1 p. 3-20, 2019. http://dx.doi.org/10.20435/inter.v0i0.1607

OLIVEIROS, B.; CARAMELO, L.; FERREIRA, N.C.; CARAMELO, F. Role of temperature and humidity in the modulation of the doubling time of COVID-19 cases. Medrxiv. 2020. https://doi.org/10.1101/2020.03.05.20031872

PREM, K. et al. The effect of control strategies to reduce social mixing on outcomes of the COVID-19 epidemic in Wuhan, China: a modelling study. The Lancet. 25 Março 2020. https://doi.org/10.1016/S2468-2667(20)30073-6

READ, J.M.; BRIDGEN, J.R.E.; CUMMINGS, D.A.T.; HO, A.; JEWELL, C.P. Novel coronavirus 2019-nCoV: early estimation of epidemiological parameters and epidemic predictions. Medrxiv. No prelo, 2020. https://doi.org/10.1101/2020.01.23.20018549

RIPAP. Rede Integrada de Pesquisa do Amapá. Carta Manifesto da Comunidade Científica Amapaense. Macapá. 2020. Disponível em: < https://drive.google.com/file/d/1Kgsikv5B27v60QPyRR6gpyEVjmnVOVfF/view >. Acesso em: 03 abr. 2020.

RC: 71076

Verfügbar in: https://www.nucleodoconhecimento.com.br/gesundheit/von-covid-19-inmacapa 
ROSA, S.J. Transporte e Exclusão Social: A Mobilidade da População de Baixa Renda da Região Metropolitana de São Paulo e Trem Metropolitano. 2006. Dissertação (Mestrado em Engenharia) - Escola Politécnica da Universidade de São Paulo, São Paulo, 2006. Disponível em: https://www.teses.usp.br/teses/disponiveis/3/3138/tde-07122006163515/publico/dissertacao_silvio_jose_rosa.pdf. Acesso em: 04 abr. 2020.

ROTHAN, H.A.; BYRAREDDY, S.N. The epidemiology and pathogenesis of coronavirus disease (COVID-19) outbreak. Journal of Autoimmunity. No prelo, 2020. https://doi.org/10.1016/j.jaut.2020.102433

ROTHE, C. et al., [Correspondece]. Transmission of 2019-nCoV Infection from an Asymptomatic Contact in Germany. Destinatário: The New England Journal of Medicine. Massachusetts, 5 de Março, 2020. https://doi.org/10.1056/NEJMc2001468

SESACE. Governo do Estado do Ceará. Secretaria de Saúde. Boletim epidemiológico: Doença pelo novo coronavírus (COVID-19) n 18. Fortaleza, CE: 2020.

SESAMA. Governo do Estado do MaranhãO. Secretaria de Saúde. Nota 9 - SES monitora 205 casos suspeitos de COVID-19. São Luís, MA: 2020.

SESAMT. Governo do Estado do Mato Grosso. Secretaria de Saúde. Secretaria Adjunta de Atenção e Vigilância em Saúde. Nota Informativa 16 COVID-19. Cuiabá, MT: 2020.

SESAMG. Governo do Estado de Minas Gerais. Secretaria de Estado de Saúde de Minas Gerais. Subsecretaria de Vigilância em Saúde. Centro de Operações de Emergência em Saúde Pública. Boletim Informativo Diário de 20/03/2020. Belo Horizonte, MG: 2020a.

RC: 71076

Verfügbar in: https://www.nucleodoconhecimento.com.br/gesundheit/von-covid-19-inmacapa 
SESAMG. Governo do Estado de Minas Gerais. Secretaria de Estado de Saúde de Minas Gerais. Subsecretaria de Vigilância em Saúde. Centro de Operações de Emergência em Saúde Pública. Boletim Informativo Diário de 14/03/2020. Belo Horizonte, MG: 2020b.

SESAMG. Governo do Estado de Minas Gerais. Secretaria de Estado de Saúde de Minas Gerais. Subsecretaria de Vigilância em Saúde. Centro de Operações de Emergência em Saúde Pública. Informe Epidemiológico n³, de 03 de Março de 2020. Belo Horizonte, MG: 2020c.

SESARS. Governo do Estado do Rio Grande do Sul. Secretaria de Saúde. Centro Operações de Emergências Rio Grande do Sul. Informe Epidemiológico - COVID19 EM 19/03/2020. Porto Alegre, RS: 2020.

SILVA, D.R.; VIANA, V.P.; MÜLLER, A.M.; LIVI, F.P.; DALCIN, P.T.R. Respiratory viral infections and effects of meteorological parameters and air pollution in adults with respiratory symptoms admitted to the emergency room. Influenza and others respiratory viruses. v. 8 n. 1, 2013. https://doi.org/10.1111/irv.12158

TORMAN, V.B.L.; COSTER, R.; RIBOLDI, J. Normalidade de variáveis: métodos de verificação e comparação de alguns testes não-paramétricos por simulação. Revista HCPA. Porto Alegre, v. 32, n. 2, p. 227-234, 2012.

TRAVASSOS C.; OLIVEIRA E.X.G.; VIACAVA F. Desigualdades geográficas e sociais no acesso aos serviços de saúde no Brasil: 1998 e 2003. Ciência \& Saúde Coletiva. v. 11 n. 4 p. $975-986$, 2006. https://doi.org/10.1590/S141381232006000400019

VELAVAN, T.P.; MEYER, C.G. The COVID-19 epidemic. Tropical Medicine \& International Health. v. 25, n.3, 2020. https://doi.org/10.1111/tmi.13383

RC: 71076

Verfügbar in: https://www.nucleodoconhecimento.com.br/gesundheit/von-covid-19-inmacapa 
VIANA, A.L.D.; IBAÑEZ, N.; ELIAS, P. E. M. Saúde, desenvolvimento e território. 1 ed. São Paulo: Hucitec; 2009.

WANG, J.; TANG, K.; FENG, K.; LV.W. High Temperature and High Humidity $\begin{array}{lllll}\text { Reduce the Transmission of COVID-19. } & \text { SSRN. }\end{array}$ https://dx.doi.org/10.2139/ssrn.3551767

WHO. Word Health Organization. Report of the WHO-China Joint Mission on Coronavirus Disease 2019 (COVID-19). China: Word Health Organization - The Joint Mission. 2020a. Disponível em: < https://www.who.int/docs/defaultsource/coronaviruse/who-china-joint-mission-on-covid-19---final-report-1100hr28feb2020-11mar-update.pdf?sfvrsn=1a13fda0_2\&download=true >

WHO. World Health Organization. Coronavirus disease 2019 (COVID-19): Situacional Report - 62. Genebra, Switzerland. WHO: 2020b.

WHO. World Health Organization. Coronavirus disease 2019 (COVID-19): Situacional Report - 38. Genebra, Switzerland. WHO: 2020c.

WU, J.T.; LEUNG, K.; LEUNG, G.M.; Nowcasting and forecasting the potential domestic and international spread of the 2019-nCoV outbreak originating in Wuhan, China: a modelling study. The Lancet. v. 395 n. 10225 p. 689-697, 2020. https://doi.org/10.1016/S0140-6736(20)30260-9

ZASLAVSKY, R.; GOULART, B. N. G. Migração pendular e atenção à saúde na região de fronteira. Ciência e Saúde Coletiva. 2017, vol.22, n.12. https://doi.org/10.1590/1413-812320172212.03522016

ZHANG, Y. et al. The Novel Coronavirus Pneumonia Emergency Response Epidemiology Team. The epidemiological characteristics of an outbreak of 2019 novel coronavirus diseases (COVID-19) in China. China CDC Weekly. v. 2, n. x, 2020. https://doi.org/10.3760/cma.j.issn.0254-6450.2020.02.003

$\mathrm{RC}: 71076$

Verfügbar in: https://www.nucleodoconhecimento.com.br/gesundheit/von-covid-19-inmacapa 
Eingesandt: April 2020.

Genehmigt: April 2020.

RC: 71076

Verfügbar in: https://www.nucleodoconhecimento.com.br/gesundheit/von-covid-19-inmacapa 